\title{
Global Mapping of an Exo-Earth Using Sparse Modeling
}

\author{
Masataka Aizawa $^{1}$ (D), Hajime Kawahara ${ }^{2,3}$ (D), and Siteng Fan $^{4}$ (D) \\ ${ }^{1}$ Department of Physics, The University of Tokyo, Tokyo 113-0033, Japan; aizawa@utap.phys.s.u-tokyo.ac.jp \\ ${ }^{2}$ Department of Earth and Planetary Science, The University of Tokyo, 7-3-1, Hongo, Tokyo, Japan \\ ${ }^{3}$ Research Center for the Early Universe, School of Science, The University of Tokyo, Tokyo 113-0033, Japan \\ ${ }^{4}$ Division of Geological and Planetary Sciences, California Institute of Technology, Pasadena, CA 91125, USA \\ Received 2019 October 23; revised 2020 April 24; accepted 2020 April 24; published 2020 June 9
}

\begin{abstract}
We develop a new retrieval scheme for obtaining two-dimensional surface maps of exoplanets from scattered light curves. In our scheme, the combination of the L1-norm and total squared variation, which is one of the techniques used in sparse modeling, is adopted to find the optimal map. We apply the new method to simulated scattered light curves of the Earth, and find that the new method provides a better spatial resolution of the reconstructed map than those using Tikhonov regularization. We also apply the new method to observed scattered light curves of the Earth obtained during the two-year Deep Space Climate Observatory/Earth Polychromatic Imaging Camera observations presented by Fan et al. The method with Tikhonov regularization enables us to resolve North America, Africa, Eurasia, and Antarctica. In addition to that, the sparse modeling identifies South America and Australia, although it fails to find Antarctica, maybe due to low observational weights on the poles. Besides, the proposed method is capable of retrieving maps from noise-injected light curves of a hypothetical Earthlike exoplanet at 5 pc with a noise level expected from coronagraphic images from a $8 \mathrm{~m}$ space telescope. We find that the sparse modeling resolves Australia, Afro-Eurasia, North America, and South America using 2 yr observation with a time interval of one month. Our study shows that the combination of sparse modeling and multiepoch observation with 1 day or 5 days per month can be used to identify main features of an Earth analog in future direct-imaging missions such as the Large UV/Optical/IR Surveyor.
\end{abstract}

Unified Astronomy Thesaurus concepts: Exoplanet astronomy (486); Astrobiology (74); Direct imaging (387)

\section{Introduction}

One of the ultimate goals of exoplanetary science is to detect life and characterize habitable environments beyond the solar system. Recent discoveries of exoplanets in the habitable zone (HZ) provide unprecedented opportunities to characterize planetary environments that could potentially harbor life (e.g., Kane et al. 2016). Beyond the detection of planets in the HZ, a promising biomarker on such planets in the $\mathrm{HZ}$ is metabolic features, such as $\mathrm{O}_{2}, \mathrm{O}_{3}$, and $\mathrm{H}_{2} \mathrm{O}$, in transmission spectra. Notably, Benneke et al. (2019) have recently reported the discovery of water vapor in the atmosphere of an $8 M_{\oplus}$ planet in the HZ (K2-18b). This discovery triggers significant motivation for seeking further indirect or direct indications of life.

In direct imaging, an exoplanet is generally accompanied by a star, whose brightness and proximity make identification of planetary light challenging. In principle, features in scattered light can be interpreted as indications of movements of surface inhomogeneity resulting from orbital and rotational motions (Ford et al. 2001). Despite the technical challenges of directly imaging Earthlike exoplanets, such a characterization of photometric variations is an important probe of exoplanetary surface environments (which may include oceans, land masses, and regions with vegetation) and planetary dynamics (Pallé et al. 2008; Cowan et al. 2009, 2011; Oakley \& Cash 2009; Fujii et al. 2010, 2011).

One of the characterization methods based on photometric variations, proposed by Kawahara \& Fujii (2010), involves two-dimensional (2D) global mapping of directly imaged planetary surfaces, using the scattered light curve in the presence of both spin and orbital motions. Subsequently, Kawahara \& Fujii (2011) formulated an inversion method named spin-orbit tomography (SOT) for recovering 2D surface maps by introducing Tikhonov regularization, which enables the direct estimation of the surface albedo. The same technique was applied successfully by Fujii \& Kawahara (2012) to reconstruct $2 \mathrm{D}$ maps, even for planets with various obliquities and orbital inclinations. Within the framework of SOT, planet obliquity is simultaneously inferred through the minimization of a loss function. The ability to infer obliquity from a light curve was extensively investigated by Schwartz et al. (2016) in terms of amplitude modulation. Also, Kawahara (2016) revealed the relation between the planet spin axis and the frequency modulation of light curves. Farr et al. (2018) constructed the Bayesian framework of 2D mapping and quantified the uncertainty in the albedo map and the obliquity. They applied the Gaussian process to regulate the interpixel variance of the map instead of the the Tikhonov regularization. On the other hand, Luger et al. (2019a) presented the open code starry that exploits spherical harmonics for mapping, and Luger et al. (2019b) applied it to the Transiting Exoplanet Survey Satellite (TESS) light curves of the Earth. Since TESS is equipped with single bandpass, the map recovered by Luger et al. (2019b) is largely affected by the cloud reflection. On the other hand, Fan et al. (2019) recently recovered a global land map by exploiting multiwavelength light curves of the Earth during the two-year Deep Space Climate Observatory/Earth Polychromatic Imaging Camera (DSCOVR/EPIC) observations (Jiang et al. 2018). Using the same data, Kawahara (2020) also reconstructed the geography and spectral components, which reasonably reproduce actual features of the Earth. These are clear practical examples of how to mitigate the effects of clouds from light curves.

The aim of this study is to improve the spatial resolution of an inferred map using sparse modeling. The global mapping is generally a ill-posed problem, so it requires regularizations for 
giving unique solutions. One possible technique is least absolute shrinkage and selection operator, which minimizes the $l_{1}$ norm simultaneously to search for the sparse solutions (e.g., Tibshirani 1996). Recently, this technique has attracted increasing attention in the field of astronomy, especially in reconstructing images from interferometric observations (e.g., Honma et al. 2014; Ikeda et al. 2016; Akiyama et al. 2017a, 2017b; Kuramochi et al. 2018; Event Horizon Telescope Collaboration et al. 2019). For example, Kuramochi et al. (2018) proposed to use the combination of total squared variations (TSV) and the $l_{1}$ norm, and they demonstrated that the technique enabled the recovery of accurate images of black hole shadow from interferometric observations compared with conventional methods.

In this paper, we apply sparse modeling to the problem of mapping planetary surfaces from reflectional light in directimaging observations. As fiducial data, we adopted the mock albedo map of the Earth and the real observational data obtained from the Deep Space Climate Observatory (DSCOVR) by Fan et al. (2019). For comparison, we solved the mapping problem using both Tikhonov regularization and sparse modeling, and discuss the difference in the output maps.

\section{Formulation of the Mapping}

\subsection{Forward Modeling of Reflected Light Curves of a Distant Planet}

For mapping the surface of an exoplanet, we estimate the planetary surface albedo $\boldsymbol{m}$, discretized to $N_{\text {pixel }}$ pixels $\left(\boldsymbol{m}=\left\{m_{j}=m\left(\theta_{j}, \phi_{j}\right)\right\}\right.$ for $\left.j=1,2, \ldots, N_{\text {pixel }}\right)$, where $\left(\theta_{j}, \phi_{j}\right)$ is the location of the $j$ th pixel on the sphere's surface. The scattered light curve $\boldsymbol{d}$ consists of $N_{\text {data }}$ points $\left(\boldsymbol{d}=\left\{d_{i}=d\left(t_{i}\right)\right\}\right.$ for $\left.i=1,2, \ldots, N_{\text {data }}\right)$, where $t_{i}$ is the $i$ th time frame. As formulated previously (e.g Kawahara \& Fujii 2010), the light curve and planetary surface are related via a transfer matrix $\boldsymbol{G}=$ $\left\{G_{i, j}=G\left(\theta_{j}, \phi_{j} ; \Phi_{i}, \Theta_{i} ; \zeta, \Theta_{\text {eq }}, i_{\text {inc }}\right)\right\} \quad$ for $i=1,2, \ldots, N_{\text {data }}$ and $j=1,2, \ldots, N_{\text {pixel }}$ as

$$
d_{i}=\sum_{j}^{N_{\text {pixel }}} G_{i, j} m_{j}+\epsilon_{i}
$$

where $\boldsymbol{G}$ describes the amount of reflection from the planetary surface toward the observer with a given influx from the central star, and $\epsilon$ corresponds to the observational uncertainties associated with $\boldsymbol{d}$. Here, $\Phi_{i}=\Phi\left(t_{i}\right)$ and $\Theta_{i}=\Theta\left(t_{i}\right)$ describe the phases of the orbital motion and spin rotation, respectively; $\zeta$ is the planetary obliquity, the angle between the planetary orbital axis and the planetary spin axis; $\Theta_{\text {eq }}$ is the orbital phase at the equinox; $i_{\text {inc }}$ is the planetary orbital inclination, which is defined as the angle between the orbital axis and the line-ofsight. In this article, we assume a circular orbit for simplicity. These geometrical quantities can be inferred from the mapping itself (e.g., Kawahara \& Fujii 2010; Schwartz et al. 2016; Farr et al. 2018) and also from the frequency modulation (Kawahara 2016). Hence, we fix $\zeta$ and $\Theta_{\text {eq }}$ to their true values for simplicity throughout this article.

Since we do not have any information in surface types, we assume isotropic (Lambertian) reflection for simplicity in this article. We note that one can also attempt to find signatures of nonisotropic reflection from light curves, which might indicate the presence of particular surface types, e.g., ocean
(Lustig-Yaeger et al. 2018). Assuming isotropic (Lambertian) reflection from the surface, $\boldsymbol{G}$ is rewritten as follows:

$$
G_{i, j} \equiv \begin{cases}\left(e_{\mathrm{S}} \cdot e_{\mathrm{R}}\right)\left(e_{\mathrm{O}} \cdot e_{\mathrm{R}}\right) \Delta \omega_{s} & \text { if } e_{\mathrm{S}} \cdot e_{\mathrm{R}}>0, e_{\mathrm{O}} \cdot e_{\mathrm{R}}>0 \\ 0 & \text { otherwise, }\end{cases}
$$

where $\Delta \omega_{s}$ is the solid angle subtended by the pixel, and $e_{\mathrm{S}}, e_{\mathrm{O}}$, and $e_{\mathrm{R}}$ are the unit vectors pointing from the discretized planetary surface specified by $\left(\theta_{j}, \phi_{j}\right)$ toward the central star, from the surface to the observer, and from the planetary center to the surface, respectively. Their expressions are given by

$$
\begin{gathered}
e_{\mathrm{S}}=\left(\cos \left(\Theta-\Theta_{\mathrm{eq}}\right), \sin \left(\Theta-\Theta_{\mathrm{eq}}\right), 0\right)^{T} \\
e_{\mathrm{O}}=\left(\sin i_{\text {inc }} \cos \Theta_{\mathrm{eq}},-\sin i_{\mathrm{inc}} \sin \Theta_{\mathrm{eq}}, \cos i_{\mathrm{inc}}\right)^{T} \\
e_{\mathrm{R}}=(\cos (\phi+\Phi) \sin \theta, \cos \zeta \sin (\phi+\Phi) \sin \theta+\sin \zeta \cos \theta \\
-\sin \zeta \sin (\phi+\Phi) \sin \theta+\cos \zeta \cos \theta)^{T} .
\end{gathered}
$$

The transfer function $\boldsymbol{G}$ has two fundamental timescales, associated with the spin and orbital periods. Combinations of these timescales allow the consideration of different positions on the planetary surface at different epochs. The inversion method exploiting this property is called SOT (see more detailed discussions in Kawahara \& Fujii 2010, 2011; Fujii \& Kawahara 2012).

\subsection{Inverse Modeling of Reflected Light Curves of a Distant Planet Using Regularization Terms}

The mapping problem to infer $m$ from $d$ in Equation (1) generally becomes ill-posed for two reasons: (a) the possible existence of invisible faces of planets seen from an observer depending on geometry; (b) angular resolution for mapping is basically limited by visible and illuminated areas for each observational snapshot, so it is not possible to reconstruct the planetary surface at infinite resolution. The first property can be mitigated by limiting the solution space not to include invisible areas, but the second one is still inevitable. There are several methods to give an unique solution to an ill-posed problem, and one of these techniques is an introduction of regularization terms to a standard chi-squared value in minimization. Previously, Kawahara \& Fujii (2011) introduced the Tikhonov regularization, also known as ridge regression, to determine the unique solution.

Although it successfully gives the unique solution, the Tikhonov regularization is not the only choice of regularization. By choosing appropriate regularization parameters, one can exploit unique features of planetary surfaces in directimaging observations: (a) the surface is composed of several major types, possibly with a few dominant species e.g., ocean in the case of the Earth; (b) the surface is likely to be continuous and smooth; (c) coastlines, on the other hand, are likely to be sharp, and they divide different surface types clearly. In this article, we attempt to exploit the first two features by introducing the L1-norm and TSV, and we evaluate the difference between the map recovered by the Tikhonov regularization and the new regularization. We also attempt other types of regularization terms including total variation, which can exploits property (c), in Appendix A, and we find that the combination of the L1-norm and TSV is likely to give the best estimation on the map. 


\subsubsection{Modeling with Tikhonov Regularization}

To solve $\boldsymbol{m}$, Kawahara \& Fujii (2011) introduced the Tikhonov regularization:

$$
\begin{gathered}
Q_{\lambda} \equiv \chi^{2}+\lambda^{2}|\boldsymbol{m}-\hat{\boldsymbol{m}}|^{2}, \\
\chi^{2}=\sum_{i=1}^{N_{\text {data }}} \frac{\left(d_{i}-\sum_{j=1}^{\left.N_{\text {pixel }} G_{i, j} m_{j}\right)^{2}}\right.}{\sigma_{i}^{2}},
\end{gathered}
$$

where $\sigma_{i}$ is the $i$ th observational uncertainty and $\hat{\boldsymbol{m}}$ is the model prior that is the uniform mean albedo map estimated from the observation (Kawahara \& Fujii 2011). The regularization parameter $\lambda$ is arbitrary, and it balances the observational noise and the spatial resolution; the large value of $\lambda$ is likely to return the uniform map similar to the prior map. The above equation can be analytically solved in the following form:

$$
\begin{gathered}
\boldsymbol{m}_{\mathrm{est}, \lambda}=V \Sigma_{\lambda} U^{T}(\tilde{\boldsymbol{d}}-\tilde{G} \hat{\boldsymbol{m}})+\hat{\boldsymbol{m}}, \\
\left(\Sigma_{\lambda}\right)_{i, j}=\frac{\kappa_{i}}{\kappa_{i}^{2}+\lambda^{2}} \delta_{i, j},
\end{gathered}
$$

where $\tilde{d}_{i}=d_{i} / \sigma_{i}$, and $\tilde{G}_{i}=G_{i} / \sigma_{i}$. The matrices $V$ and $U$ are given by the singular value decomposition of $\boldsymbol{G}=U \Lambda V^{T}$, and $\kappa_{i}$ is the $i$ th eigenvalue of the diagonal matrix $\Lambda$.

The minimization with the Tikhonov regularization is equivalent to finding the maximum posterior probability in the Bayesian statistics, where the prior is imposed as the Gaussian-type function with the mean of $\hat{m}$ and the covariance matrix $\lambda^{-2} \delta_{i, j}$. The detailed discussion is shown in Appendix C in Fujii \& Kawahara (2012).

\subsubsection{Sparse Modeling}

Alternatively to Tikhonov regularization, we consider sparse modeling, which involves the combination of the L1-norm and TSV introduced in Kuramochi et al. (2018) as the regularization terms for mapping planets. Then, the loss function $Q_{l 1, \text { tsv }}$ is defined as

$$
Q_{l 1, \mathrm{tsv}} \equiv \chi^{2}+\Lambda_{l} Q_{l}+\Lambda_{\mathrm{tsv}} Q_{\mathrm{tsv}} .
$$

The loss function $Q_{l 1 \text {,tsv }}$ is composed of the chi-squared value, the L1-norm of the map $Q_{l}$, and the TSV term $Q_{\mathrm{tsv}}$ defined as follows:

$$
\begin{aligned}
& Q_{l} \equiv \sum_{i}^{N_{\mathrm{pixel}}}\left|m_{i}\right|, \\
& Q_{\mathrm{tsv}} \equiv \sum_{i}^{N_{\text {pixel }}} \sum_{j}^{N_{\text {pixel }}} \frac{1}{2} W_{i, j}\left(m_{i}-m_{j}\right)^{2} \text {, }
\end{aligned}
$$

where $W_{i, j}$ is the neighboring matrix defined as

$$
W_{i, j}= \begin{cases}1 & \text { if } i \text { th and } j \text { th pixels are adjacent. } \\ 0 & \text { otherwise. }\end{cases}
$$

In Equation (9), $\Lambda_{l}$ and $\Lambda_{\mathrm{tsv}}$ are the regularization parameters of L1 and TSV, respectively. The second term $\Lambda_{l} Q_{l}$ describes the sparsity of the solution. The larger value of $\Lambda_{l}$ gives more zerovalued pixels in the solution. The third term $\Lambda_{\mathrm{tsv}} Q_{\mathrm{tsv}}$ in Equation (9) is defined as the sum of the difference in the values of adjacent pixels. This term determines the smoothness of the solution. We solved the minimization of $Q_{l 1, \text { tsv }}$ with a monotonic variant of the fast iterative shrinking threshing algorithm (MFISTA; Beck \& Teboulle 2009a, 2009b), following Akiyama et al. (2017a) and Kuramochi et al. (2018).

For the fair comparison with the Tikhonov regularization and its solution in Equation (7), we do not adopt a nonnegative condition for $m$, but such a constraint would help to resolve the surface. One minimization for the data in Section 3 takes a few minutes to finish, and the computational cost is scaled as $\mathcal{O}\left(\max \left(N_{\text {pixel }}^{2}, N_{\text {pixel }} N_{\text {data }}\right)\right)$. The minimization with the L1-norm and TSV term is also the same as finding the maximum posterior probability, where the prior is imposed as the combination of a Laplace distribution for the L1-norm and the Gaussian-type function for TSV.

\subsection{Choices of Regularization Parameters in Inverse Modeling}

The optimization of regularization parameters is an important issue in statistical methods, but the general discussion for the selection is difficult. In the global mapping problem with the Tikhonov regularization, Kawahara \& Fujii (2011) proposed to use the $l$-curve method, which determines the optimal solution as the maximum curvature point of the model norm versus residuals corresponding to $\chi^{2}$ (Hansen 2010). Here, the l-curve method searches for the point with the maximum curvature between $\log \mid \tilde{d}-\tilde{G} m_{\mathrm{est},\left.\lambda\right|^{2}}$ and $\log \left|m_{\text {est }, \lambda}-\hat{m}\right|$, and the detailed description of the method is found in Appendix E in Fujii \& Kawahara (2012).

In the case of sparse modeling with the combination of the L1-norm and TSV, however, the $l$-curve method cannot be applied straightforwardly because there is one additional regularization term. One might be able to extend $l$-curve to the higher-order method, but this is beyond the current scope of this work as there is no such previous study to our knowledge. Another possibility is the cross validation method, where the data are split into training and validation data, and the trained model is evaluated against the test data. However, this method favors the overfitted solution in the global mapping as discussed in Appendix B.

One possible strategy is to train regularization parameters by simulating several configurations, and apply the trained optimal regularization parameter to the real data (e.g., Event Horizon Telescope Collaboration et al. 2019). In the case of global mapping, we first prepare different land distributions, simulate the observational data for the models, recover the maps from the simulated data, and choose the acceptable regularization parameters by comparing the recovered maps with the ground truth images. Finally, one will recover the map from the real data by adopting the regularization parameters, which are determined by the injection and recovery tests in the above.

Given this procedure in mind, we focus on studying the potential of the sparse modeling in this paper; specifically, we attempt to investigate whether possible combinations of regularization parameters exist in sparse modeling that recover a better map than the Tikhonov regularization. For that, we compute a weighted residual sum of squares (WRSS) between the recovered maps and the ground map in order to identify the best map from the observation:

$$
\mathrm{WRSS}=\sum_{i}^{N_{\mathrm{pixel}}} \bar{G}_{i}\left(m_{\mathrm{est}, i}-m_{\mathrm{true}, i}\right)^{2},
$$

where the $\bar{G}_{i}$ is the time-averaged weight at the $i$ th pixel, $m_{\text {est }, i}$ is the estimated model for the map, and $m_{\text {true }, i}$ is the true map. For regions without much visibility, the term $\bar{W}_{i}$ suppresses the 
(a) Input map

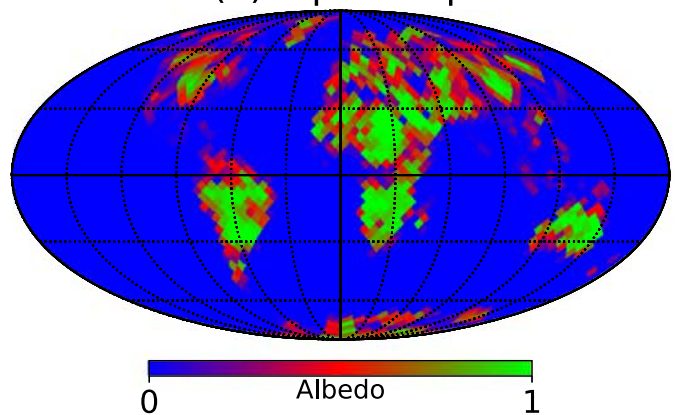

(c) Tikhonov: $\mathrm{S} / \mathrm{N}=100$

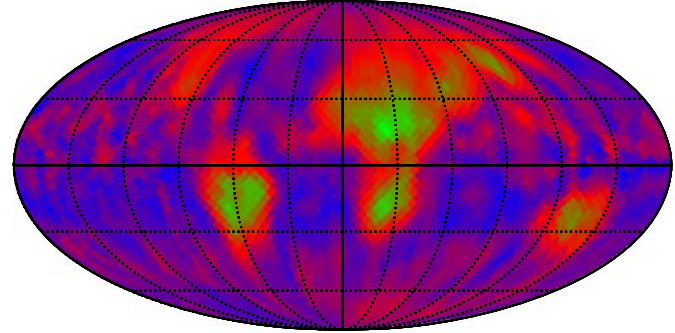

$-0.2729$

(e) Tikhonov: $\mathrm{S} / \mathrm{N}=5$

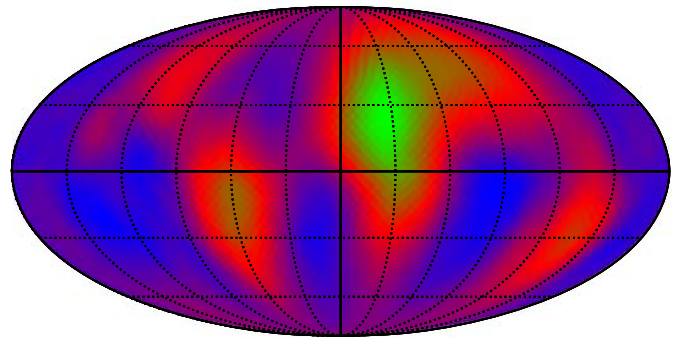

$-0.1216 \quad$ Albedo 0.7375

(g) Tikhonov: $\mathrm{S} / \mathrm{N}=2$

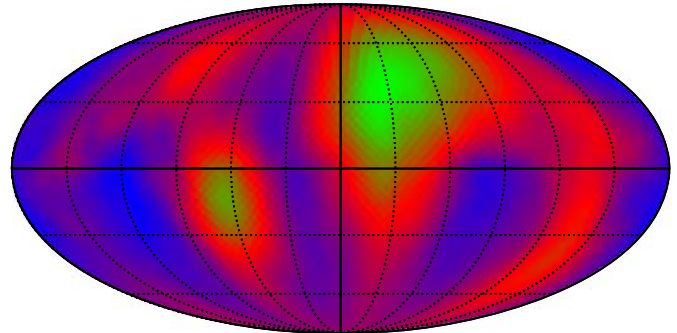

$-0.1238$

Albedo

0.6388 (b) Mean observational weight

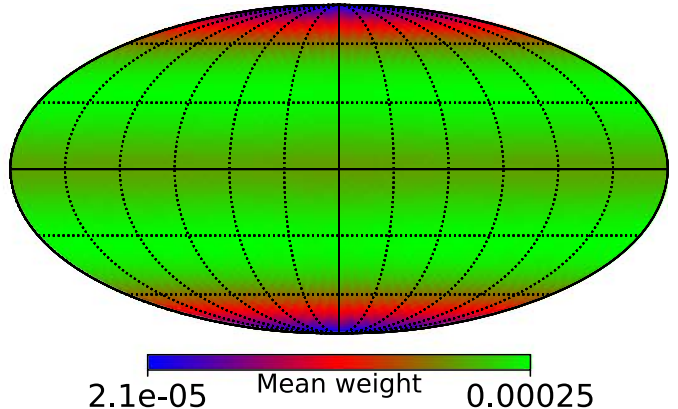

(d) Sparse modeling: $\mathrm{S} / \mathrm{N}=100$

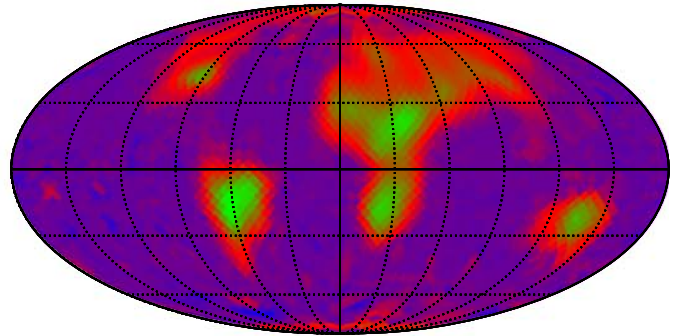

$\begin{array}{lll}-0.2797 & \text { Albedo } & 1.099\end{array}$

(f) Sparse modeling: $\mathrm{S} / \mathrm{N}=5$

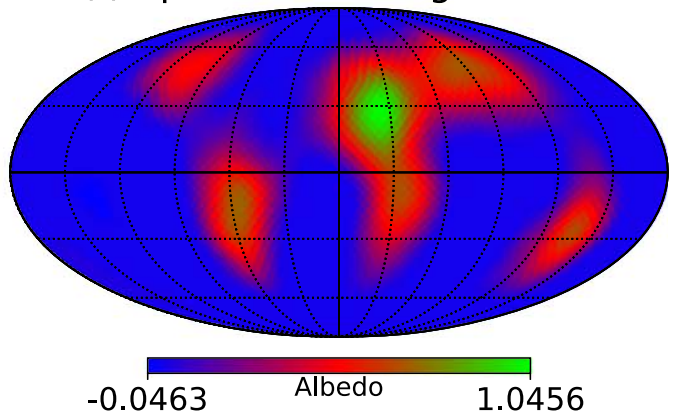

(h) Sparse modeling: $\mathrm{S} / \mathrm{N}=2$

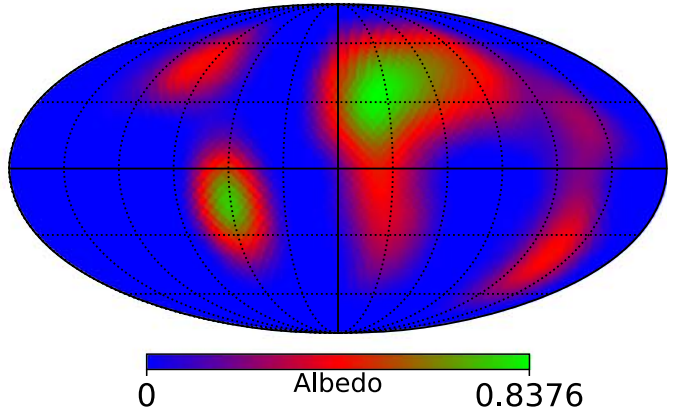

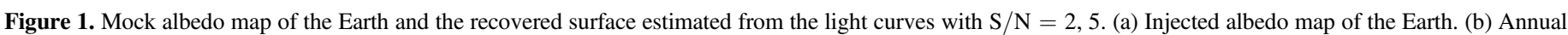

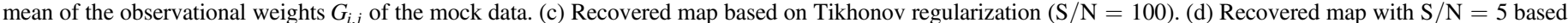

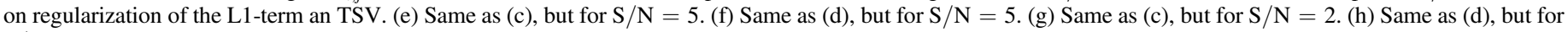
$\mathrm{S} / \mathrm{N}=2$.

degree of discrepancy between the recovered and true maps in choosing the optimal map.

\section{Mapping the Cloudless Earth}

As a test bed, we adopt a static cloud-subtracted Earth model, as used in Kawahara (2016). Figure 1(a) shows this injected albedo map of the Earth after removal of the cloud-cover fraction with ISCCP D1 data (the cloud map of
2008 June 30 21:00). On this map, the ocean has zero albedo and land has a constant albedo after subtracting the cloud coverage. The spherical pixelization was realized using Hierarchical Equal Area isoLatitude Pixelation of a sphere (HEALPix; Górski et al. 2005) with 3072 pixels in total. With regard to the TSV terms, we calculated $W_{i, j}$, referring to the orders of pixels on the sphere. Concerning the geometry and orbital parameters, we assumed $i_{\text {inc }}=0^{\circ}, \zeta=90^{\circ}, \Theta_{\text {eq }}=180^{\circ}, P_{\text {spin }}=23.93447 \mathrm{hr}$, and $P_{\text {orb }}=$ 365.24219 days. We generated a one-year light curve with 
1024 points at an $\simeq 8 \mathrm{hr}$ interval, and we added Poisson noise to the light curves by varying the signal-to-noise ratio $(\mathrm{S} / \mathrm{N})=$ 2, 5, and 100. In the analysis, we adopted "Tikhonov" and "L1-norm+TSV" regularization terms respectively for comparison. Figure 1(b) shows the mean weight of $G_{i, j}$ over the Earth's surface in this mock observation. The Earth's surface was globally surveyed except for the regions very close to the North and South Poles.

Figures 1(c)-(f) shows the recovered maps estimated from the light curves with $\mathrm{S} / \mathrm{N}=2,5$, and 100 with the "Tikhonov" and "L1-norm+TSV" regularization terms. The chosen regularization parameters are $\lambda=10^{0.1}$ for $\mathrm{S} / \mathrm{N}=2, \lambda=10^{0.3}$ for $\mathrm{S} / \mathrm{N}=5$, and $\lambda=10^{0.3}$ for $\mathrm{S} / \mathrm{N}=100$, according to the l-curve criterion (Hansen 2010) for Tikhonov regularization. For "L1-norm+TSV" regularization, we set $\left(\Lambda_{l}, \Lambda_{\text {tsv }}\right)=$ $\left(10^{-0.50}, 10^{-0.25}\right)$ for $\mathrm{S} / \mathrm{N}=2,\left(\Lambda_{l}, \Lambda_{\mathrm{tsv}}\right)=\left(10^{-0.25}, 10^{-0.25}\right)$ for $\mathrm{S} / \mathrm{N}=5$, and $\left(\Lambda_{l}, \Lambda_{\mathrm{tsv}}\right)=\left(10^{0.75}, 10^{0.25}\right)$ for $\mathrm{S} / \mathrm{N}=100$ as the optimal solutions by finding the minimum values of WRSS.

Both methods generally succeed in recovering the major continents. In case of $\mathrm{S} / \mathrm{N}=100$, both methods give wellresolved maps, there is no large difference between them, although the sparse modeling still gives the better fitting. On the other hand, as is evident from the comparison with the input map, Figures 1(d) and (f) show better resolved and more consistent maps than Figures 1(c) and (e); the detailed structures of the continents (e.g., the shapes of South America and Africa) are well reproduced in Figures 1(d) and (f). This tendency is more clearly seen in the comparison with $\mathrm{S} / \mathrm{N}=2$. The Tikhonov regularization (Figure 1(e)) fails to discriminate North and South America or Eurasia and Australia. In contrast, sparse modeling (Figure 1(f)) successfully distinguishes these continents with well-characterized coastlines. We note that the smaller $\lambda$ value gives a higher resolution with Tikhonov regularization, albeit with an overfitted inferred map and induced noise.

In addition to the case of $i_{\text {inc }}=0^{\circ}$ and $\zeta=90^{\circ}$, we also attempt $i_{\text {inc }}=45^{\circ}$ and $\zeta=23^{\circ} .4$ to estimate the surfaces by exploiting simulated light curves with $\mathrm{S} / \mathrm{N}=2,5$, and 100 . We find that $\lambda=10^{0.429}$ returns the optimal maps determined by the $l$-curve method for each case in the Tikhonov regularization. For sparse modeling, $\left(\Lambda_{l}, \Lambda_{\mathrm{tsv}}\right)=\left(10^{-1.25}, 10^{-0.50}\right)$ in the case where $\mathrm{S} / \mathrm{N}=2,\left(\Lambda_{l}, \Lambda_{\mathrm{tsv}}\right)=\left(10^{-1.00}, 10^{-0.50}\right)$ in the case where $\mathrm{S} / \mathrm{N}=5$, and $\left(\Lambda_{l}, \Lambda_{\mathrm{tsv}}\right)=\left(10^{0.25}, 10^{-0.25}\right)$ in the case where $S / N=100$. Figure 2 shows the recovered optimal maps obtained from minimizing WRSS for each case. In this setup, the Northern Hemisphere of the Earth is preferably surveyed, and most of the Southern Hemisphere is invisible due to the geometry. The sparse modeling successfully resolves the continents, especially in case of $\mathrm{S} / \mathrm{N}=2$ and 5. This example shows that the sparse modeling can work in different geometrical configurations.

Differences among the reconstructed maps originate from various aspects. Tikhonov regularization is not physically motivated by the nature of the planetary surface. This term acts as a regulator for observational noise and the spatial resolution of the surface (Kawahara \& Fujii 2011). In contrast, the L1norm efficiently identifies ocean regions because their albedo is zero. In addition, the TSV term suppresses the emergence of the bumpy structures on the maps, and the recovered maps become smoothed as a result of the minimization.

\section{Application to Observed Light Curves}

Recently, Fan et al. (2019) reproduced the surface map of the Earth using real light-curve observations of $\sim 10,000$ DSCOVR/EPIC frames collected over a two-year period. Since the DSCOVR spacecraft is located at the first Sun-Earth Lagrange point (L1), the current configuration corresponds to $\left(i_{\text {inc }}, \zeta\right)=\left(90^{\circ}, 23^{\circ} .4\right)$ with varying $\Theta_{\text {eq }}$ for $e_{\mathrm{O}}$ in Equation (3) to be parallel from the Sun to the Earth. Observations were taken in 10 optical narrowband channels, and the principal components (PCs) were calculated among all the light curves to extract the surface features. By exploiting the gradient boosting decision tree, Fan et al. (2019) found that the second strongest principal component (PC2) traces the surface inhomogeneity of planets. In particular, Fan et al. (2019) demonstrated that the time series of PC2 are linearly correlated with those of the overall land fraction, which is the summation of the land fraction weighted by $G$ viewed from the observatory at each phase. Here, the land fraction is taken from the Global Selfconsistent, Hierarchical, High-resolution Geography Database (Wessel \& Smith 1996), and it is shown in Figure 3(a). In Fan et al. (2019), they recovered the land map from PC2 using the Tikhonov regularization.

We analyze the same data as used in Fan et al. (2019). We check that our analysis with the singular value decomposition analysis of multiband observations gives the same PC2 as presented in Fan et al. (2019). For consistency, we adopt their normalized weight matrix, where the summation of weight at one epoch is equal to one, slightly different from the current definition of $\boldsymbol{G}$. Figures 3(b) and (c) show the mean weight matrix of the observation obtained by EPIC on board DSCOVR and the corresponding recovered map, respectively. This observation is fairly insensitive to the North and South Poles because DSCOVR is always located near the first Lagrangian point between the Earth and the Sun. In their solution, they adopted $\lambda=10^{-1.5}$ as the regularization parameter in Equation (5). Their estimation (Figure 3(c)) captured the coarse features of the Earth's surface, but not all the continents were successfully recovered (e.g., South America and Australia).

For comparison, we solved the same problem using the L1norm and TSV regularization terms. The light curves have negative and positive values owing to the nature of the principal component. In the solution derived by Fan et al. (2019), the regions with negative PC2 are more likely to be ocean. To make efficient use of sparsity, i.e., to associate zero values to ocean regions, we produced the nonnegative light curves by subtracting the minimum value of the light curves from the overall light curves, and slightly offsetting the entire data set by 0.005 . This ad hoc operation, or the choice of the added constant, does not significantly affect the results because the constant offset in the light curve would only result in a constant change to the whole recovered map. Figure 3(d) shows the recovered map, based on the L1-norm and TSV regularization. For a clear comparison, we convert the PC2 of the recovered maps into a corresponding land fraction by using the relation, $\mathrm{PC} 2=0.0753 \times$ land fraction -0.0214 , which is derived from the linear regression. In the minimization, we assume the associated noise $\sigma_{i}=1$ for simplicity. We adopted $\left(\Lambda_{l}, \Lambda_{\mathrm{tsv}}\right)=\left(10^{-3}, 10^{-3}\right)$ in Equation (9) as the optimal solution chosen by minimizing WRSS between the recovered map and the land fraction of the Earth in Figure 3(a). Notably, the mapping by the new regularization term resolves the 
(a) Input map

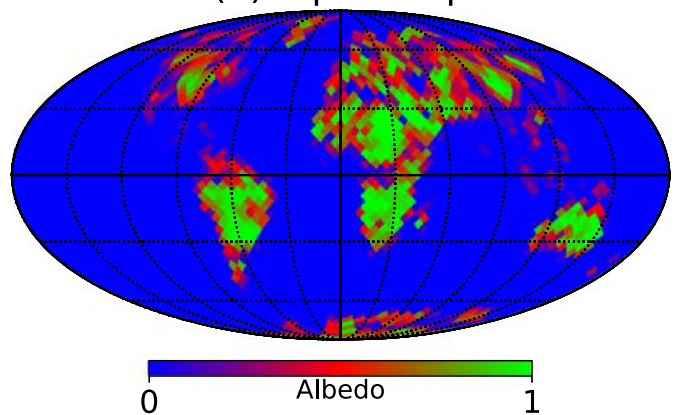

(c) Tikhonov: $\mathrm{S} / \mathrm{N}=100$

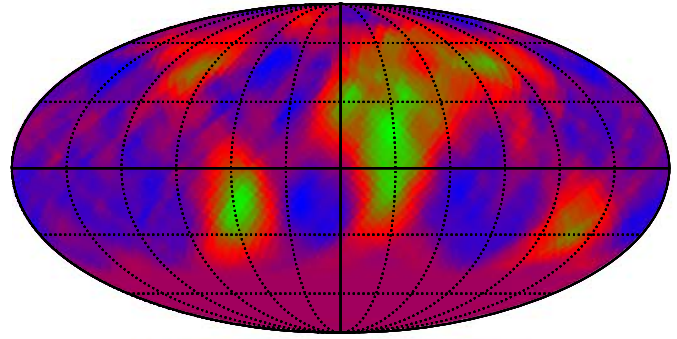

$-0.2095$

(e) Tikhonov: $\mathrm{S} / \mathrm{N}=5$
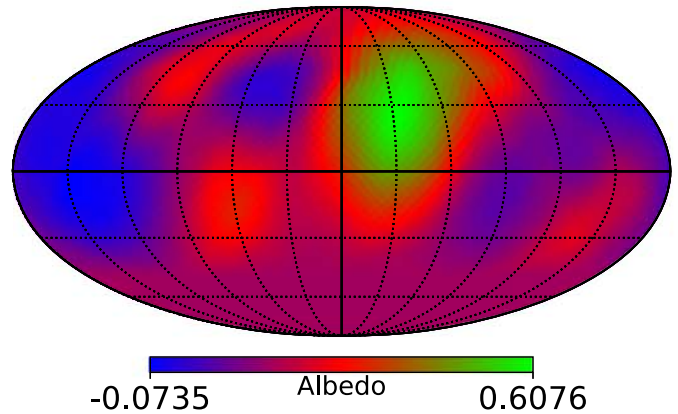

(g) Tikhonov: $\mathrm{S} / \mathrm{N}=2$
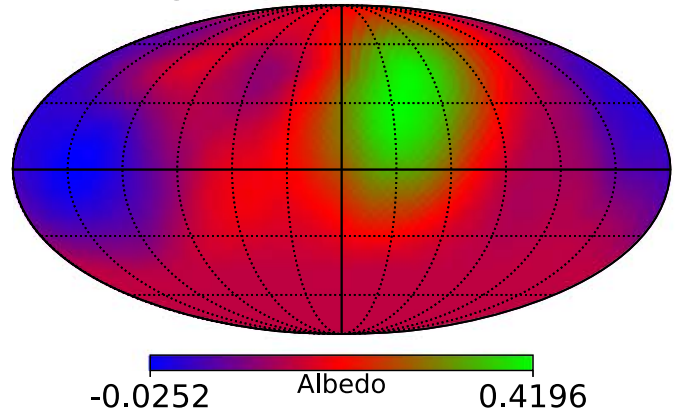

(b) Mean observational weight

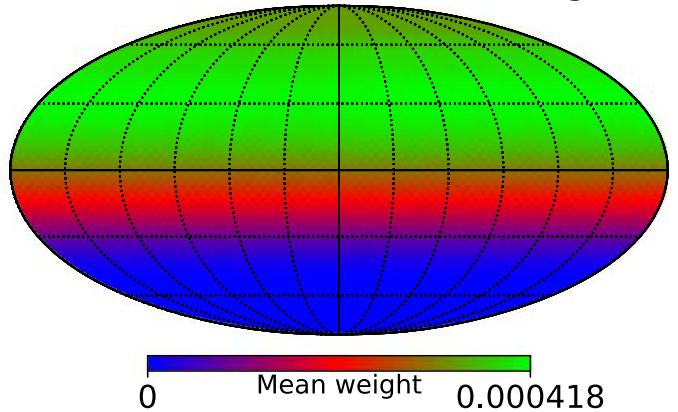

(d) Sparse modeling: $\mathrm{S} / \mathrm{N}=100$

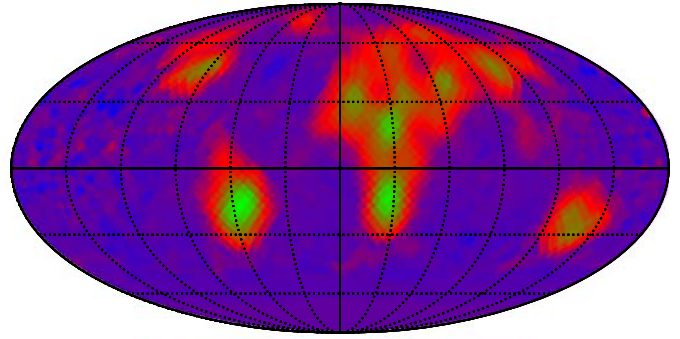

$-0.2443 \quad$ Albedo 1.1911

(f) Sparse modeling: $\mathrm{S} / \mathrm{N}=5$

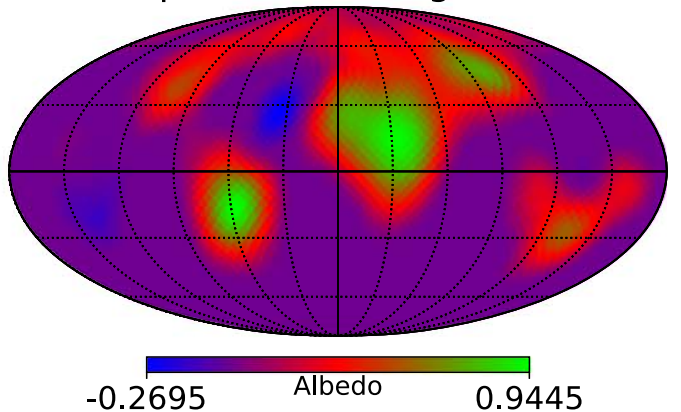

(h) Sparse modeling: $\mathrm{S} / \mathrm{N}=2$

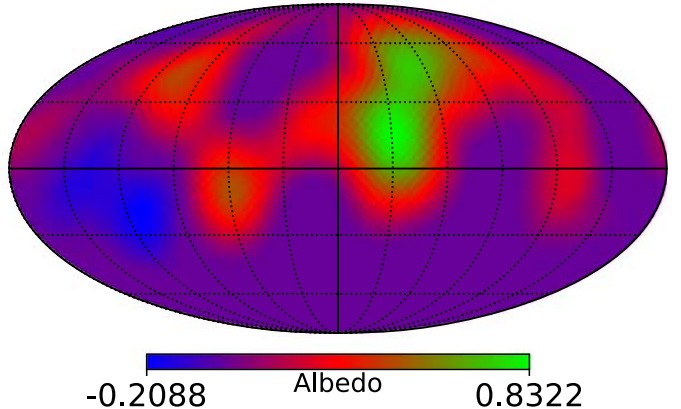

Figure 2. Same as Figure 1, but for $\zeta=23.445$ and $i=45^{\circ}$.

structure of South America and Australia, which are blurred and connected to Antarctica in Figure 3(c). It also successfully resolves other continents very consistently with the features of the Earth surface. However, our newly-proposed map does not recover Antarctica as implied in Figure 3(c). This may be due to its low observational weights (Figure 3(b)) and different spectral features than other continents.

Finally, we show solutions with nine different combinations of $\Lambda_{l}=\left(10^{-4}, 10^{-3}, 10^{-2}\right)$ and $\Lambda_{\mathrm{tsv}}=\left(10^{-4}, 10^{-3}, 10^{-2}\right)$ in Figure 4. Toward the larger value of $\Lambda_{l}$, the reconstructed maps become more sparse, and inconsistent with the ground truth.
On the other hand, with a smaller value of $\Lambda_{l}$, the solutions become more similar to those obtained from the Tikhonov regularization (Figure 3(c)). The optimal solution with $\left(\Lambda_{l}, \Lambda_{\mathrm{tsv}}\right)=\left(10^{-3}, 10^{-3}\right)$ is exactly in the middle of these two kinds of solutions, and the three cost functions in $Q_{l 1 \text {,tsv }}$ balance each other at this point.

\section{Demonstration of Global Mapping in Future Observations}

In future space direct-imaging observations (e.g., the Habitable Exoplanet Observatory (HabEx) and the Large 
(a) Land map of the Earth

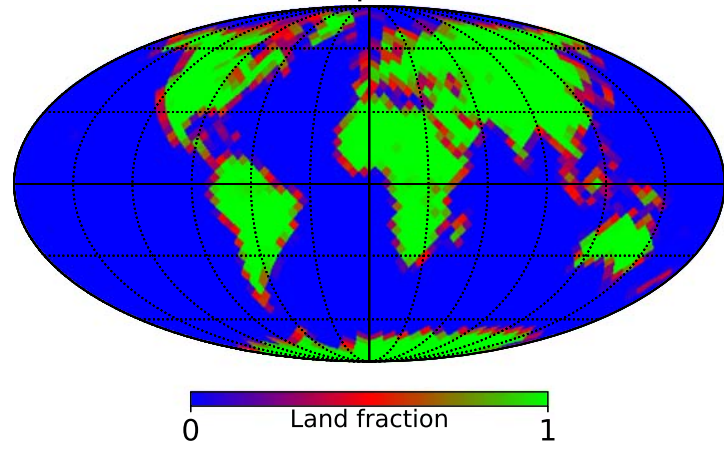

(c) Tikhonov

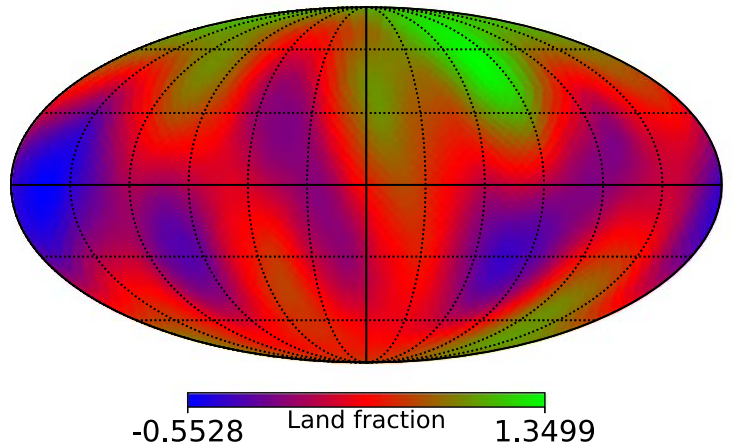

(b) Mean observational weight

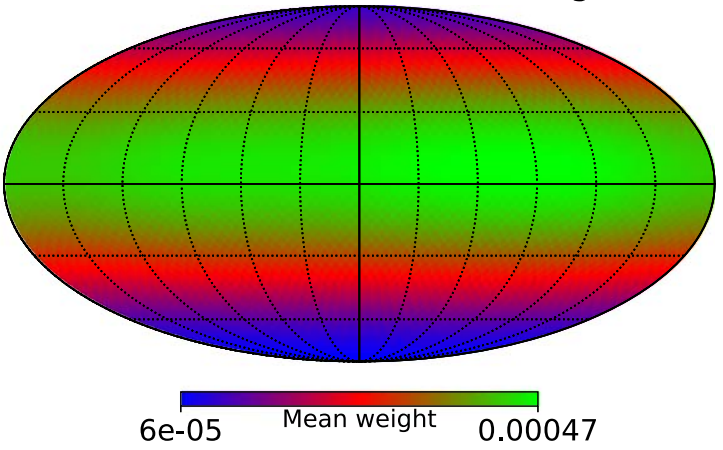

(d) Sparse modeling

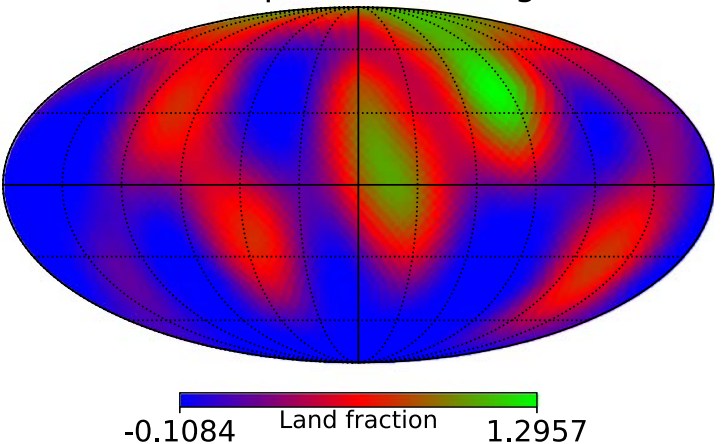

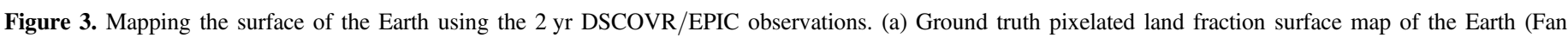

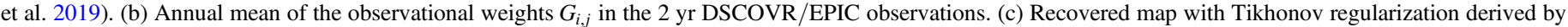

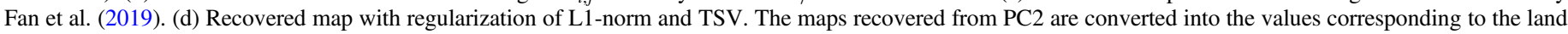
fraction.

UV/Optical/IR Surveyor (LUVOIR)), it would be unrealistic to have continuous occupancy of an instrument for years. In addition, the instrumental and astrophysical noise in actual exoplanet observations would be orders of magnitude larger than those in Earth's light curves. It is necessary to take the consideration of many fewer time frames and a higher noise level for exoplanet observations. In this section, we test the feasibility of the method under a more realistic assumption.

As a fiducial system, we consider an Earth-sized planet around a Sun-like star with $T=5780 \mathrm{~K}\left(T_{\odot}\right)$ and $R_{\star}=R_{\odot}$ at a distance of $5 \mathrm{pc}$. The semimajor axis of the planetary orbit is assumed to be $1 \mathrm{au}$, and the planetary flux in each band is calculated by convolving the planet's reflectivity with the stellar spectrum. For simplicity, we assume the reflection to be Lambertian, and the phase angle $\alpha$ is assumed to be $90^{\circ}$, where the planet is at a quadrature. We consider a LUVOIR-like telescope with a diameter $D=8 \mathrm{~m}$. The coronagraph design contrast is assumed to be $10^{-10}$, and the end-to-end throughput in the coronagraph is taken to be $0.3 .^{5}$ The integration time is $1.8 \mathrm{hr}$, which corresponds to the average time interval in the current observation. For simplicity, we consider the snapshot of each image rather than the smeared image with $1.8 \mathrm{hr}$ integration. However, if we take into account the smearing effect, the recovered image would be blurred by $27^{\circ}$, which corresponds to $1.8 \mathrm{hr}$, around the spin axis. Although the current cadence is already limited by the

\footnotetext{
5 The expected coronagraph contrast and end-to-end throughput of the ECLIPS instrument is adopted from the LUVOIR Final Report (http://www. latex-cmd.com/struct/footnote.html). The average optical throughput in the visible band is assumed to be 0.277 in the imaging mode.
}

observational strategy of DSCOVR, one will be able to evade this problem by adopting a shorter cadence.

We calculate the observational noises using coronagraph, which is an open source Python package for computing the noise of space direct-imaging missions (Robinson et al. 2016; Lustig-Yaeger et al. 2019). Adopting the imaging mode in coronagraph, we compute $\mathrm{S} / \mathrm{N}$ in each band. As the DSCOVR observation uses narrowband filters $(317,325,340$, $388,443,552,680,688,764$, and $779 \mathrm{~nm})$, we reassign the band centers and FWHM to emulate broadband filters of our mock observatory. For instance, we take the average of the light curves in three narrowband filters of DSCOVR $(317,325$, and $340 \mathrm{~nm})$ as the light curve in a single band. In the similar manner, we combine the light curves in the filters with band centers of 680 and $688 \mathrm{~nm}$, and 764 and $779 \mathrm{~nm}$, by averaging their light curves. As a result, we have the light curve with six broadband filters with band centers $=325,388,443,552,684,770 \mathrm{~nm}$ and $\mathrm{FWHM}=30,33,22,87,45,41 \mathrm{~nm}$.

Figure 5 shows the compositions of the noise sources based on Robinson et al. (2016). The calculation is basically based on the sample code "luvoir_demo.py" in coronagraph. The noise sources are composed of Poisson noise, local zodiacal light, exozodiacal light, dark current, read noise, and speckle noise. In the current case, the dominant noise sources are Poisson noise and speckle noise. In the reconstructed broadband filters, we find $\mathrm{S} / \mathrm{N}=10.50,15.17,15.13,26.92,16.18$, 15.40 assuming $1.8 \mathrm{hr}$ integration.

Instead of a continuous $2 \mathrm{yr}$ observation, we assume multiepoch observations like those presented in Schwartz et al. (2016) and Farr et al. (2018). We divide $1 \mathrm{yr}$ into 


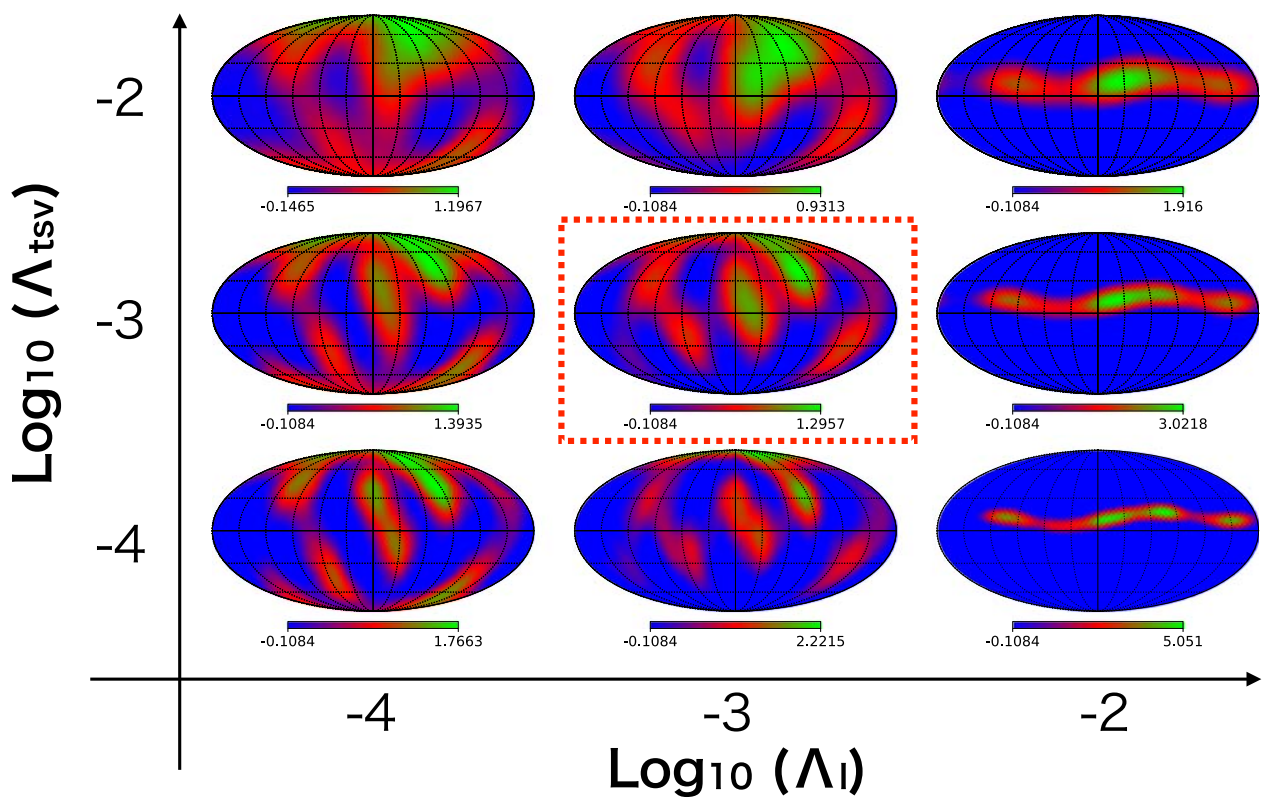

Figure 4. Recovered surface maps with different combinations of $\Lambda_{l}=\left(10^{-4}, 10^{-3}, 10^{-2}\right)$ and $\Lambda_{\mathrm{tsv}}=\left(10^{-4}, 10^{-3}, 10^{-2}\right)$. The optimal solution is shown in the center of the panel being surrounded by red dotted lines.

(a) Coronagraph noise

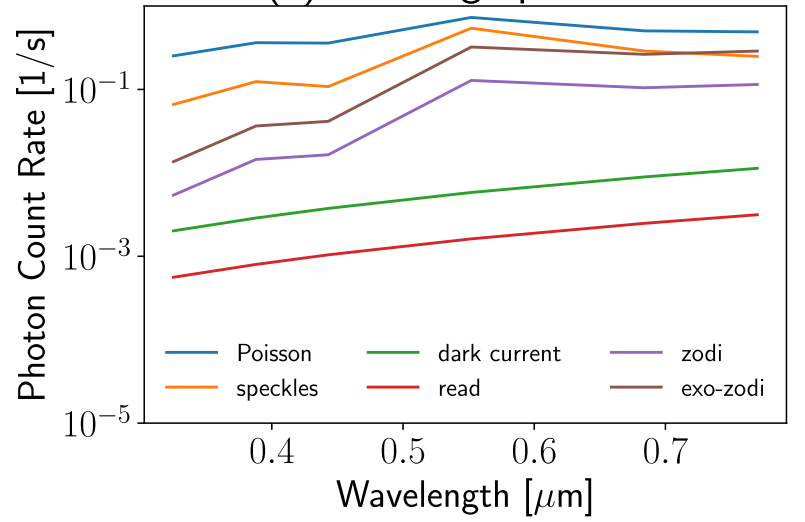

(b) Correlation b/w PC2 and Land fraction $\left(D_{\mathrm{obs} / \mathrm{month}}=5\right.$ days $)$

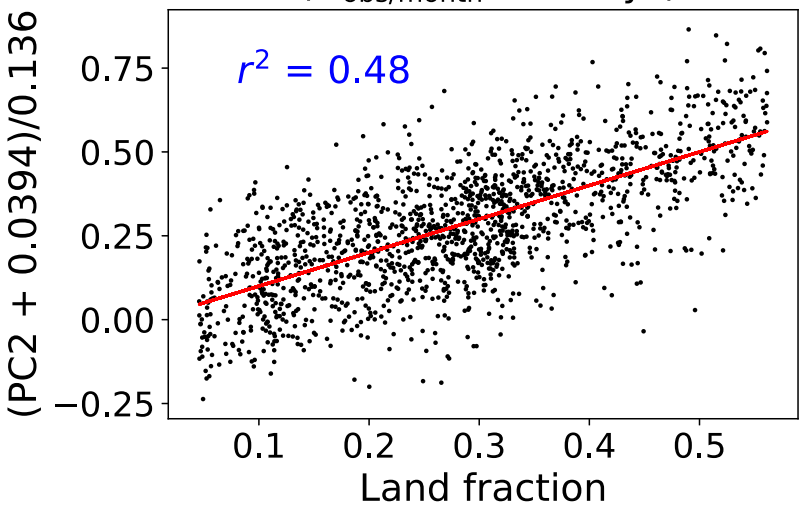

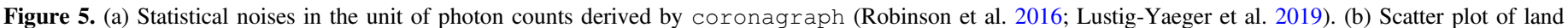

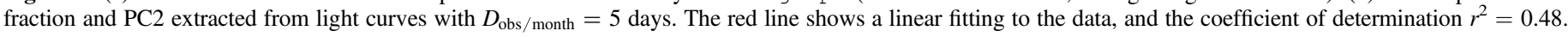

12 blocks ( $\simeq 1$ month), and use the first $D_{\text {obs } / \text { month }}=1$ or 5 days in each block. Adopting $\mathrm{S} / \mathrm{N}$ as above, we inject Gaussian noise to the light curves, and decompose them into principal components using the singular value decomposition (SVD). As revealed in Fan et al. (2019), the second strongest principal component (PC2) is linearly correlated with the land fraction; the coefficient of determination $r^{2}$ is 0.91 in their paper. Figure 5 shows the scatter plot of PC2 and the land fraction in case of $D_{\text {obs } / \text { month }}=5$ days with $r^{2}=0.48$, which implies the weak correlation. Similarly, we find $r^{2}=0.48$ for $D_{\text {obs } / \text { month }}=1$ day and $r^{2}=0.50$ for the full data set. On the other hand, we find $r^{2}=0.93$ without the injection of noise, so the noise would account for the weak correlation in the current case. We fit a linear function of the land fraction to the $\mathrm{PC} 2$, and we find PC2 $=0.290 \times$ land fraction -0.083 for $D_{\mathrm{obs} / \text { month }}=1$ day, and PC2 $=0.136 \times$ land fraction -0.039 for $D_{\text {obs } / \text { month }}=5$ days. The coefficients roughly differ by a factor of $\sqrt{5}$ between $D_{\text {obs } / \text { month }}=1$ and 5 days, and this is because the normalization of PC2 is equal to 1 by definition. Finally, we adopt (PC2 + $0.083) / 0.290$ for $D_{\text {obs } / \text { month }}=1$ day and $(\mathrm{PC} 2+0.039) / 0.136$ for $D_{\text {obs } / \text { month }}=5$ days for solving the map in order for $\boldsymbol{m}=0$ to correspond to zero land fraction i.e., ocean.

Figure 6 shows the recovered surface maps using Tikhonov regularization and sparse modeling for $D_{\mathrm{obs} / \text { month }}=1$ and 5 days, respectively. We express the recovered maps in representation of land fraction by exploiting the linear relation for PC2, and the optimal solutions are obtained by minimizing WRSS between the recovered maps and the land map of the Earth in Figure 3(a) for both Tikhonov regularization and sparse modeling. Notably, even in the case of $D_{\text {obs } / \text { month }}=$ 1 day, the planetary surface is roughly resolved in the Tikhonov regularization. Moreover, the sparse modeling (Figure 6(b)) successfully resolves Australia, Afro-Eurasia, North America, and South America although it is still blurred. On the other hand, observations with $D_{\text {obs/month }}$ of 5 days in Figure 6 enable us to recover the maps with very similar accuracy to those produced from continuous observations. These results 


\section{Global Mapping of Exo-Earth}

(a) Tikhonov reguralization

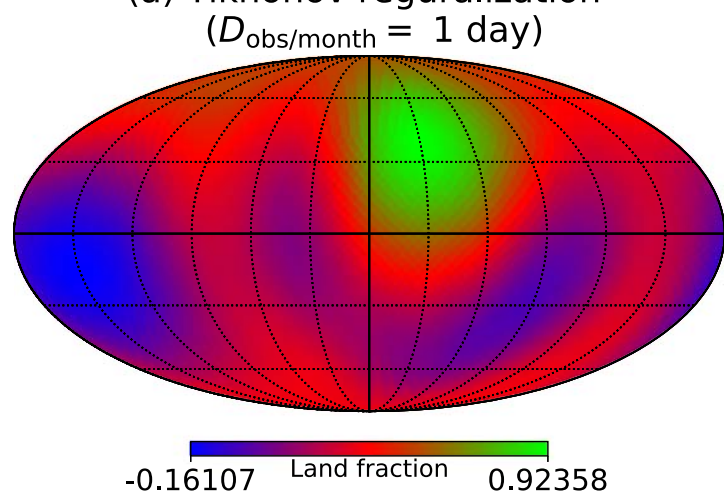

$-0.16107$

(c)Tikhonov reguralization

$\left(D_{\text {obs } / \text { month }}=5\right.$ days $)$

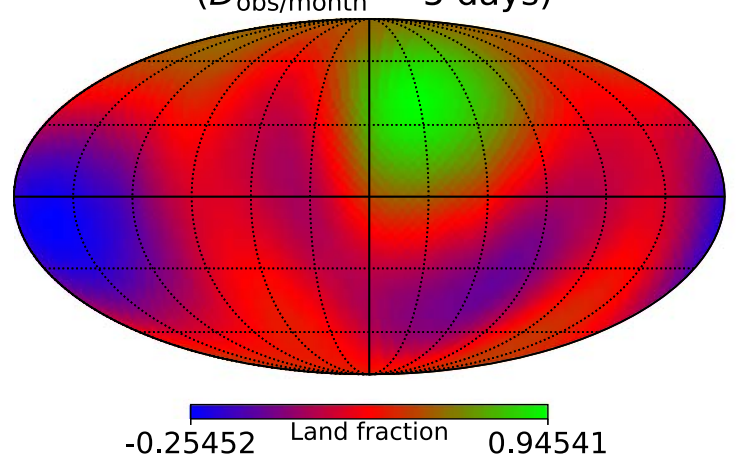

(b) Sparse modeling

$\left(D_{\text {obs } / \text { month }}=1\right.$ day $)$

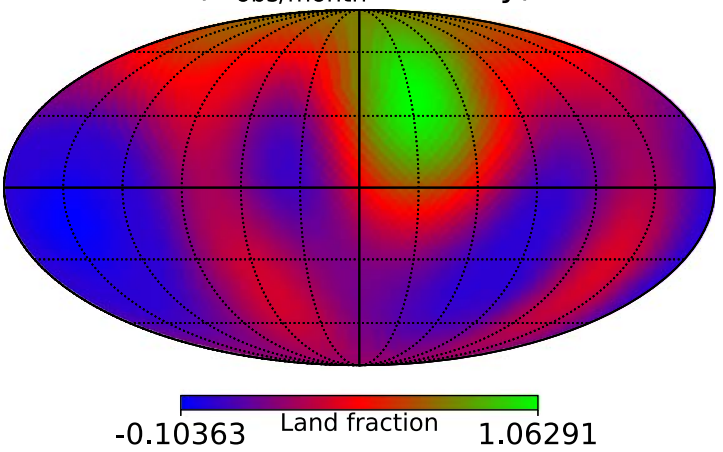

(d) Sparse modeling

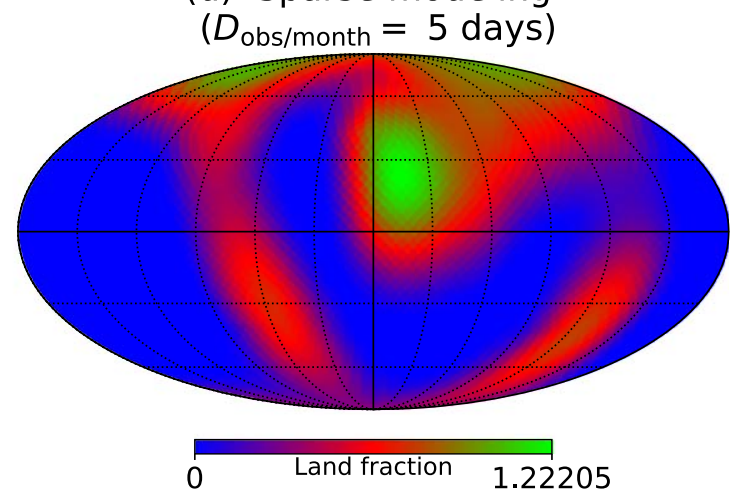

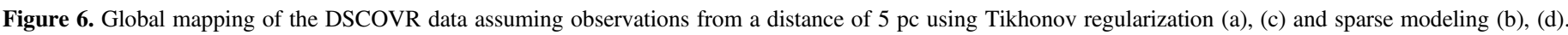

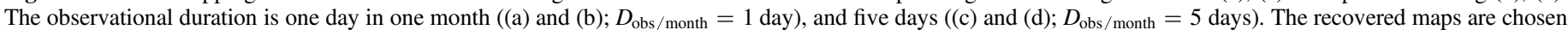
by minimizing WRSS between the recovered maps and the land map of the Earth.

encourage the mapping of "Second Earth" using future directimaging missions such as HabEx and LUVOIR.

\section{Summary}

The use of reflected light curves for mapping surfaces is an important probe of exoplanet surface inhomogeneity. Previous studies of recovering surface maps from observed light curves basically regularized observational noise with Tikhonov regularization without the consideration of physical properties of planetary surfaces (e.g Kawahara \& Fujii 2011; Fujii \& Kawahara 2012; Farr et al. 2018; Farr et al. 2018; Kawahara 2020). In addition to those studies with pixel discretization on the sphere, Luger et al. (2019b) exploited the spherical harmonics functions for solving the mapping problem using the TESS light curve of the Earth, and they adopted priors, which suppress high order features of the surface. In this paper, we introduced sparse modeling (L1+TSV regularization) to estimate a planetary surface efficiently by exploiting the following surface properties: (a) distinct spectral difference between land and ocean and (b) continuity of planetary surfaces. As a test calculation, we injected and recovered the mock albedo map of the Earth using both regularization terms, and found that sparse modeling reproduces more consistent continental surface distributions than the Tikhonov regularization. We also applied our method to observations of the Earth obtained by DSCOVR to investigate the advantage of sparse modeling in real data analysis. We found that sparse modeling successfully recovers the continents (Australia and South America) that are not clearly estimated by Tikhonov regularization. We also showed that 1 or 5 days per month in a $2 \mathrm{yr}$ observation would enable us to retrieve the surface map of an Earth analog at a distance of $5 \mathrm{pc}$.

In this paper, we demonstrate that the choice of regularization terms in exoplanet mapping can significantly affect the resulting recovered maps. The regularization terms can be seen as the priors from a Bayesian viewpoint, and our study shows the importance of selecting regularizations or priors for finding the correct map from the observation. In this viewpoint, the discretization on the planetary surface rather than the spherical harmonics would be intuitive to put reasonable priors that exploit characteristics of planetary surfaces. We also attempt other types of regularization terms, and the combination of L1+TSV regularization is likely to be the best choice among possible regularizations in case of the Earth. In the current sparse modeling, we make use of the sparsity coming from the very low albedo of the ocean, but even for a planet without seas, we can apply the method by offsetting the data to force an albedo of a particular surface type to be zero. In this paper, we consider only the case of the Earth, and other continental configurations, geometries, regularization terms, and the optimal choice of the regularization parameter for unknown surfaces are subject to future investigations. 
The authors thank the DSCOVR team for making the data publicly available. We also thank Yasushi Suto and Shiro Ikeda for fruitful discussions on sparse modeling and its application for mapping, and we thank Yuk L. Yung for discussions on surface map retrieval using DSCOVR observations. This work is supported by JSPS KAKENHI Grant Nos. 14J07182 (M.A.), JP17K14246, JP18H04577, JP18H01247, and JP20H00170 (H.K.). M.A. is also supported by the Advanced Leading Graduate Course for Photon Science (ALPS) and by the JSPS fellowship. This work was also supported by the JSPS Core-toCore Program "Planet" ${ }^{2 "}$ and SATELLITE Research from Astrobiology center (AB022006).

\section{Appendix A Comparison of Different Regularizations}

In this section, we compare six different regularizations in a mapping problem. For that purpose, we assume the same condition as done in Section 3: $\mathrm{S} / \mathrm{N}=5, i_{\text {inc }}=0^{\circ}$, and $\zeta=90^{\circ}$. By comparing the recovered maps from simulated light curves, we demonstrate that the L1+TSV is likely to be the best regularization for the mapping problem.

For comparison, we introduce additional regularization, total variation (TV), defined as

$$
Q_{\mathrm{tv}} \equiv \sum_{i}^{N_{\text {pixel }}} \sum_{j}^{N_{\text {pixel }}} \frac{1}{2} W_{i, j}\left|m_{i}-m_{j}\right|,
$$

where we exploit the same neighboring matrix $W_{i, j}$ as used for the TSV term. We define the regularization parameter for TV as $\Lambda_{\mathrm{TV}}$. The above expression cannot be differentiable when $m_{i}=m_{j}$, so we use replace $\left|m_{i}-m_{j}\right|$ by $\sqrt{\left(m_{i}-m_{j}\right)^{2}+\epsilon^{2}}$, where $\epsilon=10^{-8}$ in this study, when we compute the derivative. With the TV term, we can search for solutions with sparsity in derivatives of the surface albedo in the current problem. Since the planetary surface is roughly divided into several types with boundaries, the TV term possibly helps to identify the clear edges.

Using this new term, we exploit six different regularizations in total for estimating the planetary surface: L1+TSV, TV, L1 + TV, TSV, Tikhonov regularization, and L1-norm. To find the optimal solutions, we implement a grid search, where the ranges of the regularization parameters are from $10^{-3}$ to $10^{1}$. The optimal regularization parameters are $\left(\Lambda_{1}, \Lambda_{\text {tsv }}\right)=$ $\left(10^{-0.25}, 10^{-0.25}\right)$ for $\mathrm{L} 1+\mathrm{TSV}, \quad \Lambda_{\mathrm{tv}}=10^{-0.5}$ for $\mathrm{TV}$, $\left(\Lambda_{\mathrm{l}}, \Lambda_{\mathrm{tv}}\right)=\left(10^{0.56}, 10^{-0.33}\right)$ for $\mathrm{L} 1+\mathrm{TV}, \Lambda_{\mathrm{tsv}}=10^{0.25}$ for TSV, $\lambda=10^{0.30}$ for Tikhonov regularization, and $\Lambda_{1}=$ $10^{-0.5}$ for L1-norm. Figure A1 shows the comparison of recovered maps with six different regularizations, where L1 +TSV and Tikhonov regularization are already studied in Section 3. The maps are determined by minimizing WRSS in Equation (12) from comparison with the input model of the Earth. Consistent with the expectation, the TV term separates the different regions well by making clear boundaries between them, but it also tries to connect the continents by introducing new layers, which should be oceans in reality, at the same time. This is also the case for the combination of L1+TV. On the other hand, in case of the L1-norm alone, the anomalously high albedo values are associated with small pieces on the surface, unlike the real distribution of the Earth's surface. In the future observation of exoplanets with direct imaging, a planetary radius and albedo can be degenerate, and the L1-norm would misunderstand the exo-Earth as a very large planet, whose reflectivity mostly comes from small regions. In summary, we find WRSS $=0.0249,0.0271,0.0282,0.0286,0.0292$, and 0.626 for $\mathrm{L} 1+\mathrm{TSV}, \mathrm{TV}, \mathrm{L} 1+\mathrm{TV}$, TSV, Tikhonov regularization, and L1-norm, respectively. Therefore, L1+TSV is the best choice among the current possibilities. 
(a) Input map

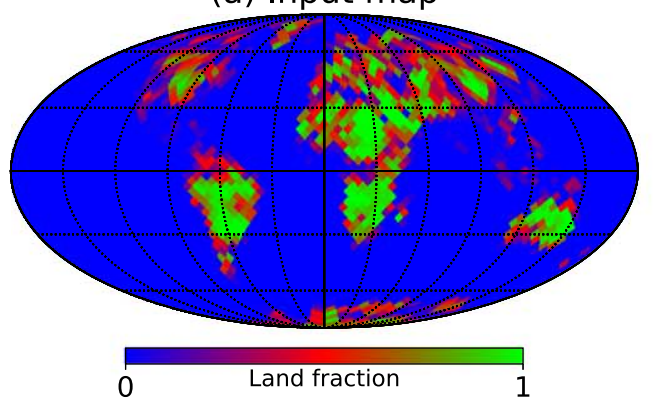

(c) L1 regularization

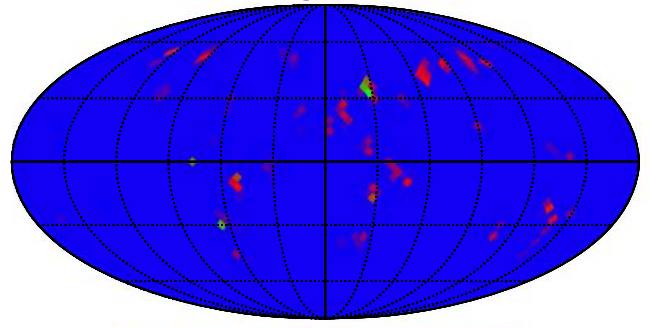

$-0.476$

(e) TSV regularization

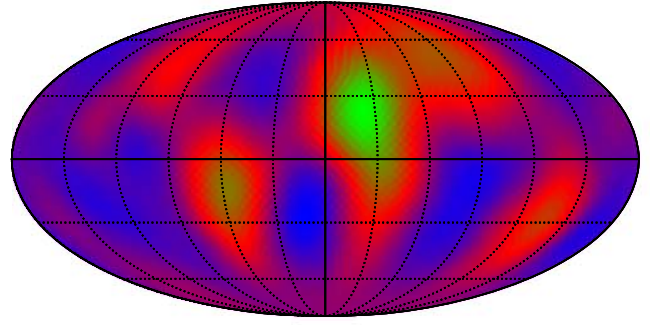

-0.189 Albedo $\quad 0.859$

(g) L1 + TSV regularization

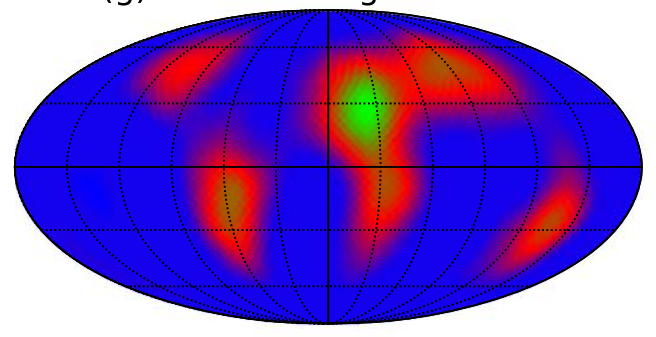

$-0.046$

Albedo

1.046 (b) Observational converage

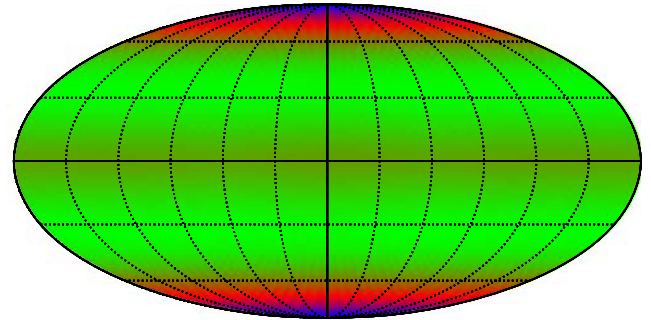

2.1e-05 Observational weight 0.00025

(d) TV regularization

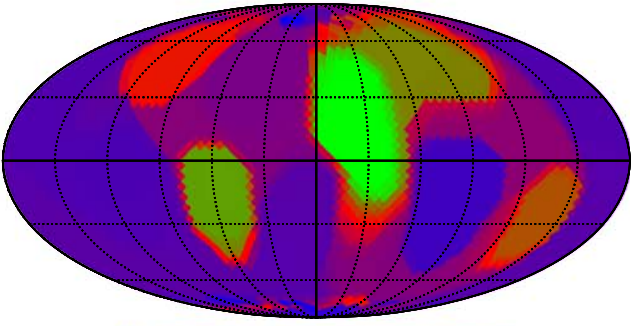
$-0.134$
Albedo
0.668

(f) Tikhonov regularization
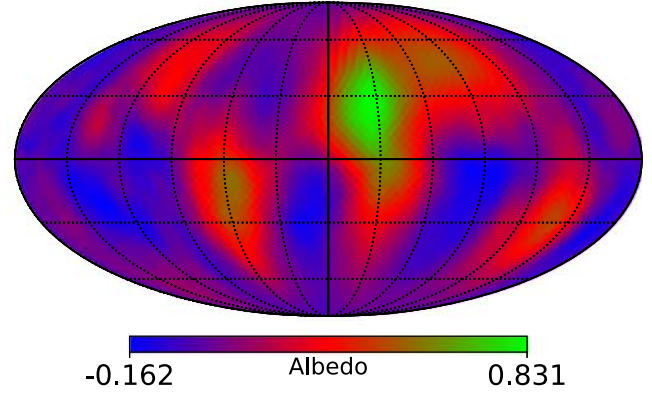

(h) L1 + TV regularization

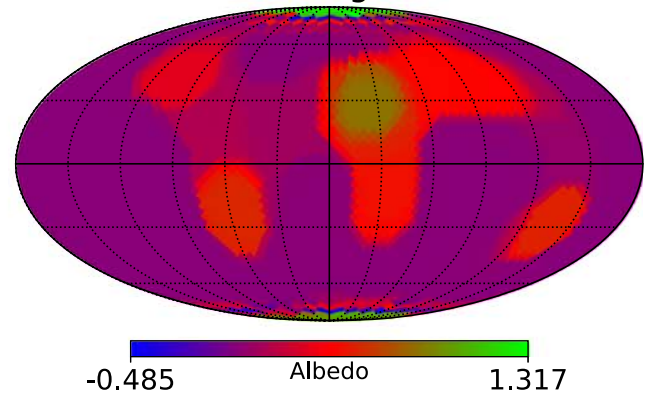

Figure A1. Comparison of recovered maps in different methods $\left(\mathrm{S} / \mathrm{N}=5\right.$ ) (a) Injected albedo map of the Earth. (b) Annual mean of the observational weights $G_{i, j}$ of the mock data. (c) Recovered map based on L1 regularization. (d) Recovered map based on TV regularization. (e) Recovered map based on Tikhonov regularization. (f) Recovered map based on L1+TSV regularization. (g) Recovered map based on L1+TV regularization.

\section{Appendix B \\ Cross Validation, $l$-curve Method, and Comparison with Ground Truth}

In this section, we compare the cross validation and $l$-curve method for recovering the maps in the Tikhonov regularization. Here, the cross validation is a statistical method, in which we split the data into subsets, perform training using the data except for one test subset, and assess the goodness of the trained model by comparing its prediction with the remaining test data. In this article, we adopt tenfold cross validation to obtain the root mean squared error (RMSE). Specifically, we divide the original light curve into 10 random subsets, select one subset as the test data, the other nine as the training data, and compute the mean squared error between the prediction and the test data. We iterate this procedure 10 times, using each subset as the test data, and compute the mean of RMSE. In the analysis, we adopt the same light-curve and geometrical configuration as used in the case of $\mathrm{S} / \mathrm{N}=5$. Figure $\mathrm{B} 1$ (a) shows the deviations of recovered maps from the ground truth map, depending on the regularization parameter $\lambda$. The blue point corresponds to the map closest to the ground truth map, the black point is obtained from the cross validation in (c), and 


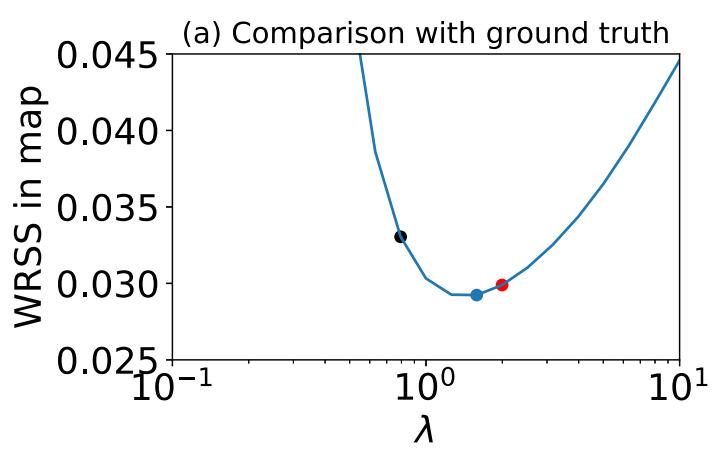

(b) Closest to ground truth

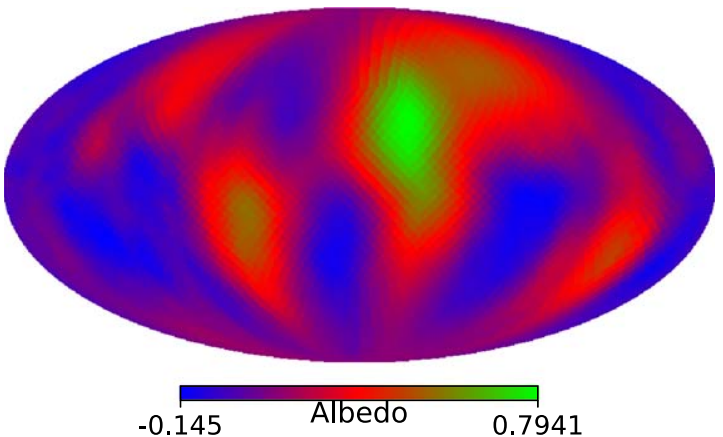

(d) Cross validation

(c) Cross validation

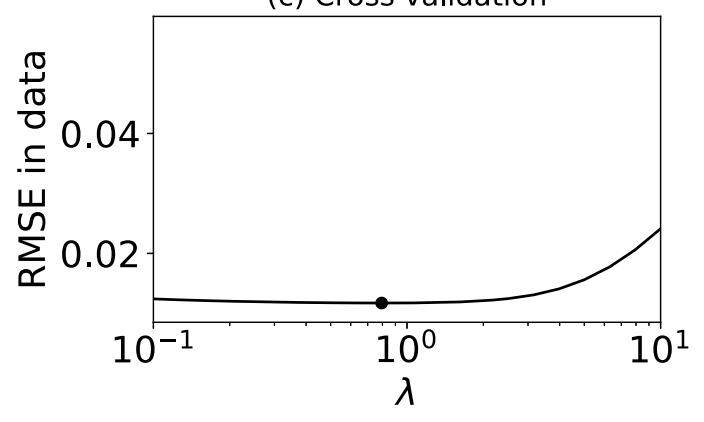

(e) I-curve method

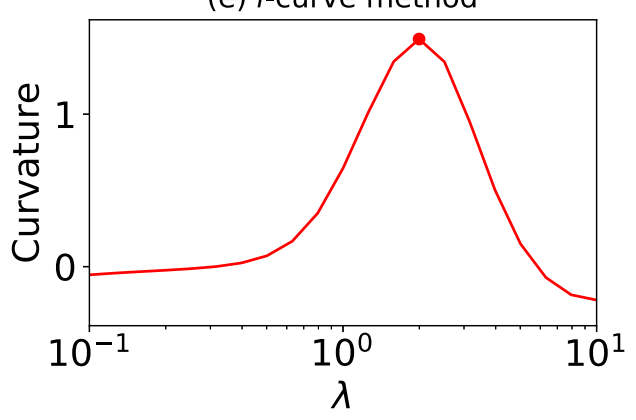

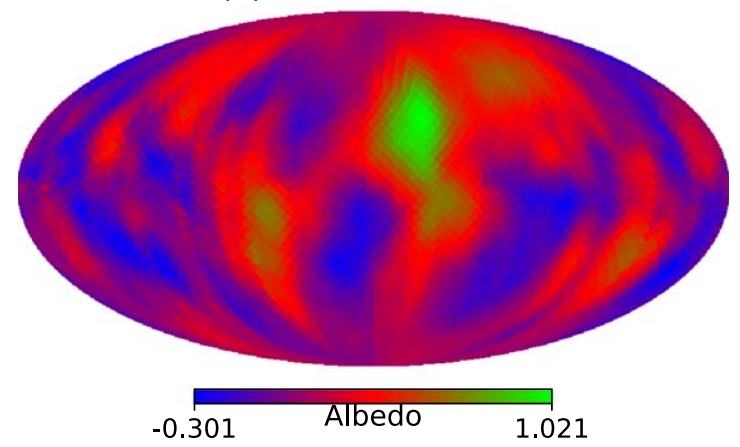

(f) l-curve method

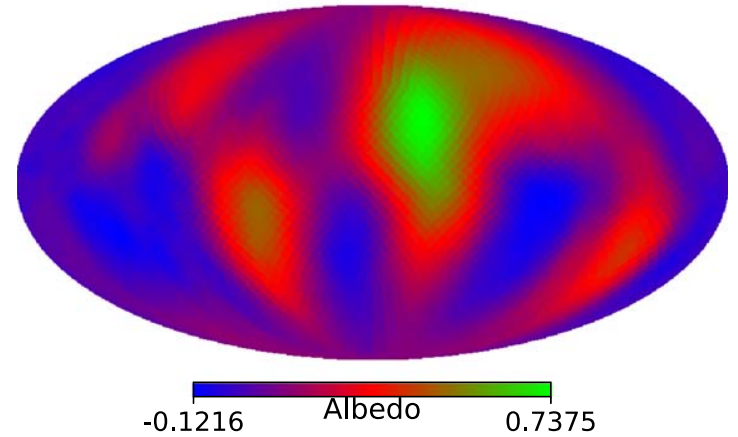

Figure B1. Comparison of recovered maps from the light curves with $\mathrm{S} / \mathrm{N}=5$ in Tikhonov regularization. Blue, black, and red points correspond to optimal maps in (b), (d), and (f), respectively. (a) RMS errors between recovered maps and the ground truth map. (b) Recovered map obtained from comparison with the ground truth map with $\lambda=10^{0.2}$. (c) RMS errors in cross validation. (d) Recovered map obtained from the cross validation with $\lambda=10^{-0.1}$. (e) Curvature exploited in the $l$-curve method. (f) Recovered map obtained from the $l$-curve method with $\lambda=10^{0.3}$.

the red point is determined by the $l$-curve method in (e). Panels (b), (d), and (f) correspond to the recovered maps obtained from the comparison with the ground truth map, the cross validation, and the $l$-curve method, respectively.

The comparison demonstrates that the cross validation gives the overfitted map, and it deviates from the ground truth map. In addition, rms errors in the cross validation are significantly insensitive to $\lambda$, implying that the method can return the very overfitted solution with reasonable rms errors. On the other hand, the $l$-curve method returns the map close to the best possible map by comparison with the ground truth map. Therefore, the $l$-curve method works at the same level of the comparison with the ground truth, although the cross validation does not.

\section{ORCID iDs}

Masataka Aizawa (D) https:// orcid.org/0000-0001-8877-4497 Hajime Kawahara (iD https://orcid.org/0000-0003-3309-9134 Siteng Fan (1) https://orcid.org/0000-0002-3041-4680

\section{References}

Akiyama, K., Ikeda, S., Pleau, M., et al. 2017a, AJ, 153, 159 Akiyama, K., Kuramochi, K., Ikeda, S., et al. 2017b, ApJ, 838, 1 Beck, A., \& Teboulle, M. 2009a, SIAM J. Imaging Sci., 2, 183 Beck, A., \& Teboulle, M. 2009b, ITIP, 18, 2419

Benneke, B., Wong, I., Piaulet, C., et al. 2019, ApJL, 887, L14 Cowan, N. B., Agol, E., Meadows, V. S., et al. 2009, ApJ, 700, 915

Cowan, N. B., Robinson, T., Livengood, T. A., et al. 2011, ApJ, 731, 76

Event Horizon Telescope Collaboration, Akiyama, K., Alberdi, A., et al. 2019, ApJL, 875, L4

Fan, S., Li, C., Li, J.-Z., et al. 2019, ApJL, 882, L1

Farr, B., Farr, W. M., Cowan, N. B., Haggard, H. M., \& Robinson, T. 2018, AJ, 156,146

Ford, E. B., Seager, S., \& Turner, E. L. 2001, Natur, 412, 885

Fujii, Y., \& Kawahara, H. 2012, ApJ, 755, 101

Fujii, Y., Kawahara, H., Suto, Y., et al. 2010, ApJ, 715, 866

Fujii, Y., Kawahara, H., Suto, Y., et al. 2011, ApJ, 738, 184

Górski, K. M., Hivon, E., Banday, A. J., et al. 2005, ApJ, 622, 759

Hansen, P. C. 2010, Discrete Inverse Problems: Insight and Algorithms, Vol. 7 (Philadelphia, PA: SIAM)

Honma, M., Akiyama, K., Uemura, M., \& Ikeda, S. 2014, PASJ, 66, 95 
Ikeda, S., Tazaki, F., Akiyama, K., Hada, K., \& Honma, M. 2016, PASJ, 68, 45 Jiang, J. H., Zhai, A. J., Herman, J., et al. 2018, AJ, 156, 26

Kane, S. R., Hill, M. L., Kasting, J. F., et al. 2016, ApJ, 830, 1

Kawahara, H. 2016, ApJ, 822, 112

Kawahara, H. 2020, ApJ, 894, 58

Kawahara, H., \& Fujii, Y. 2010, ApJ, 720, 1333

Kawahara, H., \& Fujii, Y. 2011, ApJL, 739, L62

Kuramochi, K., Akiyama, K., Ikeda, S., et al. 2018, ApJ, 858, 56

Luger, R., Agol, E., Foreman-Mackey, D., et al. 2019a, AJ, 157, 64

Luger, R., Bedell, M., Vanderspek, R., \& Burke, C. J. 2019b, arXiv:1903. 12182
Lustig-Yaeger, J., Meadows, V. S., Tovar Mendoza, G., et al. 2018, AJ, 156,301

Lustig-Yaeger, J., Robinson, T., \& Arney, G. 2019, JOSS, 4, 1387

Oakley, P. H. H., \& Cash, W. 2009, ApJ, 700, 1428

Pallé, E., Ford, E. B., Seager, S., Montañés-Rodríguez, P., \& Vazquez, M. 2008, ApJ, 676, 1319

Robinson, T. D., Stapelfeldt, K. R., \& Marley, M. S. 2016, PASP, 128, 025003 Schwartz, J. C., Sekowski, C., Haggard, H. M., Pallé, E., \& Cowan, N. B. 2016, MNRAS, 457, 926

Tibshirani, R. 1996, J. R. Stat. Soc. B, 58, 267

Wessel, P., \& Smith, W. H. F. 1996, JGR, 101, 8741 